P03.25 PERCEPTION OF HIGH SCHOOL STUDENTS ON RISK FOR ACQUIRING HIV AND UTILISATION OF VOLUNTARY COUNSELLING AND TESTING (VCT) SERVICE FOR HIV IN DEBRE-BERHAN TOWN, ETHIOPIA: A QUANTITATIVE CROSS-SECTIONAL STUDY

${ }^{1} \mathrm{~S}$ Sisay*, ${ }^{2} \mathrm{~W}$ Erku, ${ }^{2} \mathrm{G}$ Medhin, ${ }^{3} \mathrm{D}$ Woldeyohannes. ${ }^{1}$ Department of Biomedicine, School of Health Science, Dilla University, P. O. Box 419, Dilla, Ethiopia; ${ }^{2}$ Department of Tropical and Infectious Diseases, Aklilu Lemma Institute of Pathobiology, Addis Ababa University, P. O. Box 1176, Addis Ababa, Ethiopia; ${ }^{3}$ Department of Public Health, School of Medicine and Health Sciences, Addis Ababa Science and Technology University, P. O. Box 16417, Addis Ababa, Ethiopia

\subsection{6/sextrans-2015-052270.253}

Introduction Human immunodeficiency virus (HIV) epidemic among youth is largely ignored and remains invisible to both young people themselves and to the society as a whole. Thus, the aim of the study was to assess the extent of perception risk of HIV and utilisation of voluntary counselling and testing (VCT) service among high school students at Debre-berhan Town, Amhara Regional State, Ethiopia.

Methods A cross-sectional study was carried out from November 2010 up to January 2011 among secondary school students at Debre-berhan Town. Perception risk and VCT use were considered as dependant variables. A stratified random sampling technique was used to recruit study participants by taking schools as strata. Semi-structured self-administered questionnaire was used to collect the necessary data. Data were entered and analysed using SPSS version 17.0. P-value $<0.05$ was considered as statistically significant.

Results A cross-sectional study was carried out from November 2010 up to January 2011 among secondary school students at Debre-berhan Town. Perception risk and VCT use were considered as dependant variables. A stratified random sampling technique was used to recruit study participants by taking schools as strata. Semi-structured self-administered questionnaire was used to collect the necessary data. Data were entered and analysed using SPSS version 17.0. P-value $<0.05$ was considered as statistically significant.

Conclusion Some students were engaged in risky sexual behaviour even though they had heard about HIV/AIDS. The perception of risk for acquisition of HIV infection and utilisation of VCT were low. Thus, education on topic of HIV/AIDS through integrating as part of school curriculum and encouraging the existing health institutions to provide youth-friendly sexual counselling services including VCT for HIV are strongly recommended.

Disclosure of interest statement The authors declare that they have no any competing interests.

\section{P03.26 PARENTS' AND TEACHERS' VIEWS ON ADOLESCENTS' SEXUAL HEALTH TO INFORM THE DEVELOPMENT OF A SCREENING INTERVENTION FOR SEXUALLY TRANSMITTED INFECTIONS: A QUALITATIVE STUDY}

\footnotetext{
${ }^{1} \mathrm{GH}$ Wanje*, ${ }^{2} \mathrm{~L}$ Masese, ${ }^{1} \mathrm{E}$ Avuvika, ${ }^{3} \mathrm{G}$ Omoni, ${ }^{4} \mathrm{~A}$ Baghazal, ${ }^{1,2,5,6} \mathrm{RS}$ McClelland. ${ }^{1}$ University of Nairobi Institute of Tropical \& Infectious Disease (UNITID), Nairobi, Kenya; ${ }^{2}$ Department of Medicine, University of Washington, Seattle, Washington, USA; ${ }^{3}$ School of Nursing Sciences, University of Nairobi, Nairobi, Kenya; ${ }^{4}$ Mombasa County Department of Health, Mombasa, Kenya; ${ }^{5}$ Department of Epidemiology, University of Washington, Seattle, Washington, USA; ${ }^{6}$ Department of Global Health, University of Washington, Seattle, Washington, USA
}

10.1136/sextrans-2015-052270.254
Introduction In sub-Saharan Africa, communication between parents and adolescents regarding sex is often proscribed. Lack of parental communication could prevent adolescents from seeking sexual health services such as screening for sexually transmitted infections (STIs). We explored parents' and teachers' attitudes, and enlisted their help, in designing an STI screening intervention for female adolescents in Mombasa, Kenya.

Methods Between June 2013 and August 2014, we approached parents and teachers from three high schools. Purposive sampling was used to recruit willing parents and teachers to participate in-depth interviews (IDIs) and focus-group discussions (FGDs). Topics included general knowledge on STIs, reproductive health education, and willingness of parents to support their female adolescents in screening for STI. Transcripts were coded and analysed using content analysis.

Results We conducted 11 IDIs (5 parents and 6 teachers) and 4 FGDs ( 2 with parents and 2 with teachers, total of 26 participants). Most parents reported few or no discussions regarding STIs with their adolescent girls. Parents were more comfortable discussing consequences of sexual activity including loss of virginity and the potential for pregnancy. Parents tend to shift the responsibility for sexual health discourse to the teachers. The teachers, in turn, provide basic sexual and reproductive health education including abstinence, overview of STIs, and understanding of puberty, according to the educational curriculum. Both parents and teachers found the idea of screening for STIs in female adolescents to be acceptable, and were comfortable with our contacting them through informational meetings at schools. However, all parents felt that adolescents' screening results should be shared with their parents.

Conclusion Parents and teachers were supportive of a STI screening intervention, beginning with informational meetings at schools. Research and programs that aim to provide STI screening for adolescents in this setting must address the issue of whether results will be disclosed to parents.

Disclosure of interest statement This study was supported by the University of Washington Global Centre for Integrated Health of Women, Adolescents, and Children. Infrastructure and logistical support for the Mombasa Field Site was received from the University of Washington and Fred Hutchinson Cancer Research Centre's Centre for AIDS Research (grant number P30AI27757). No pharmaceutical grants were received in the development of this study.

\section{P04 - Partners, places and STI risk}

\section{P04.01 SIMILARITIES AND DIFFERENCES IN PERCEPTIONS OF MODELS FOR ONLINE PARTNER NOTIFICATION FOR SEXUALLY TRANSMITTED INFECTIONS: POTENTIAL USERS VERSUS CARE PROVIDERS}

${ }^{1} \mathrm{M}$ Doull, ${ }^{2} \mathrm{D}$ Haag*, ${ }^{2} \mathrm{M}$ Bondyra, ${ }^{3,4} \mathrm{C}$ Lee, ${ }^{5} \mathrm{~K}$ Dinner, ${ }^{5} \mathrm{~T}$ Wong, ${ }^{1,2,6} \mathrm{M}$ Gilbert. ${ }^{1}$ University of British Columbia; ${ }^{2} B C$ Centre for Disease Control; ${ }^{3}$ Simcoe Muskoka District Health Unit; ${ }^{4}$ University of Toronto; ${ }^{5}$ Public Health Agency of Canada; ${ }^{6}$ Ontario HIV Treatment Network

\subsection{6/sextrans-2015-052270.255}

Background Online partner notification (OPN) services are used by a subset of patients diagnosed with sexually transmitted infections (STI); however, less is known about their perception by care providers. We compared opinions of potential users and providers with respect to different OPN models including email 
vs text, and open (anyone can access) vs closed models (provider controls access via e-mail invitation or access code).

Methods We conducted five focus groups with potential users (youth, men who have sex with men (MSM), STI clinic clients), community agencies, and public health nurses, and interviewed family physicians, recruited through multiple methods. Participants were shown visual depictions of OPN models and examples of existing services, and opinions elicited using discussion guides that probed acceptability, advantages, and challenges of differing models. Notes taken were supplemented by review of audio recordings and analysed thematically.

Results We spoke with 16 potential users (6 youth, 6 MSM, 4 clients), 4 agency staff, 11 nurses, and 8 physicians. Older and younger users preferred OPN through e-mail and texting respectively, each perceiving the chosen modality as more serious and private. Participant points of convergence included: OPN are beneficial; need for two-stage messaging (initial generic, followed by detailed disease and contact information); few concerns regarding misuse; limitations given online sex-seeking without contact information. Most users preferred OPN for all possible STI while providers more commonly emphasised reportable or treatable infections. Users preferred closed access models which were perceived as more serious and secure. Providers preferred open models, perceiving closed models to create barriers for clients and difficult to integrate into clinical practice.

Conclusion We found overall support for OPN, but key differences between client and provider perceptions may pose challenges to uptake. As OPN are best promoted by providers giving an STI diagnosis, understanding and addressing provider concerns is important.

Disclosure of interest statement The authors have no conflicts of interest to disclose.

\section{P04.02 PARTNER NOTIFICATION IN NORTH AMERICA: A HISTORICAL ACCOUNT}

${ }^{1} \mathrm{O}$ Sobanjo, ${ }^{2} \mathrm{M}$ Steben*, ${ }^{*} \mathrm{E}$ Cheuk, ${ }^{4} \mathrm{~J}$ Kettner, ${ }^{3} \mathrm{M}$ Fast, ${ }^{4} \mathrm{H}$ Meadwick. ${ }^{1}$ Resident, Public Health and Preventive Medicine, Faculty of Medicine, McGill University, Montréal, Québec, Canada; ${ }^{2}$ STI Unit, Institut National de Santé Publique Du Québec, Montréal, Québec, Canada; ${ }^{3}$ Formerly From National Collaborative Centre on Infectious Diseases (NCCID), Winnipeg, Manitoba, Canada; ${ }^{4}$ National Collaborative Centre on Infectious Diseases, Winnipeg, Manitoba, Canada

10.1136/sextrans-2015-052270.256

Introduction Partner notification (PN) has been an integral part of sexually transmitted infection (STI) prevention for decades. Initially proposed for syphilis control, it now extends to a wide range of STI. NCCID PN project included documenting its origins in North America (NA).

Methods A multi-pronged approach has been adopted due to paucity of historical evidence.

- Literature search: electronic database (PubMed), manual reference list search, printed literature on public health $(\mathrm{PH})$ history in NA, search of significant $\mathrm{PH}$ organisation websites for related publications.

- Semi-structured interviews of key participants in STD control programs National STD prevention conference in Minnesota.

- Blogging: obtain historical accounts through use of online discussion sites or focus groups e.g. stdpreventiononline.org

- Request of materials: participants in the online blog and those for the structured interviews were to bring memorabilia, documents, letters.
Results PN might have been in use since the 19th century, PN has been shown effective when implemented as part of a larger $\mathrm{PH}$ initiative. Crucial to its success was the monitoring of performance. Confidentiality was a priority. Identifiers were used in reports to make it possible to trend networks and patterns. PN evolved in time with changes in diseases. Efforts were made to make available free services for testing and treatment. The costs implications favoured adoption of methods such as providerreferral and patient-referral. Given the importance of PN to STI prevention, it was disappointing to find limited documentation published particularly in Canada compared to United States.

Conclusion PN has been touted as the cornerstone in STI control. Looking at its origins and principles of success helps informs decisions regarding its current role and practice. Prevailing circumstances such as restricted budgets, high rates of mobility and increasing numbers of anonymous partners call for a modification in the use of PN today. Newer models employing social media are being developed.

Disclosure of interest statement The National Collaborating Centre for Infectious Diseases is funded by the Public Health Agency of Canada. No pharmaceutical grants were received in the development of this study.

\section{P04.03 WHICH PARTNER NOTIFICATION METHOD DO PATIENTS PREFER? RESULTS OF A PATIENT PREFERENCE SURVEY AT THE NATIONAL STI CLINIC IN SINGAPORE}

WS Tan*, TW Chio. Department of STI Control Clinic, Singapore

\subsection{6/sextrans-2015-052270.257}

Introduction Partner Notification (PN) can be made more effective if source patients are receptive to the method of PN. With increased mobile and internet connectivity, we carried out a survey to find out if our patient's preference towards PN had changed.

Methods A self-administered survey was carried out on clinic attendees over 4 weeks in February 2014. Respondents were asked about their preference for each PN method on a modified 4 point Likert scale.

Results A total of 416 completed survey forms were collected. The demographics of the respondents were consistent with the average clinic demographic: $74 \%(n=307)$ were male, $26 \%(n$ $=109$ ) were female. $71.6 \%$ were heterosexual, $23.6 \%$ were homosexual, and $4.8 \%$ bisexual. $90.5 \%$ had access to an Iphone/ Android or both.

If diagnosed with an STI, respondents rated the following methods as good/very good: Self notification (84.3\%), Phone call by clinic $(52.4 \%)$, SMS from clinic $(46.4 \%)$, Email sent by clinic (29.6\%), Letter sent by clinic (22.3\%).

If their partners had an STI, respondents rather the following PN methods as good/very good: Self notification (88.7\%), Phone call by clinic (54.1\%), SMS from clinic $(48.8 \%)$, Email sent by clinic (29.5\%), Letter sent by clinic (21.9\%).

Subgroup analyses found that significantly more respondents aged below 32 years preferred SMS $(52.6 \%$ vs. $42.2 \%, \mathrm{p}=$ 0.039 ). MSM respondents significantly preferred email as a PN method compared to heterosexual respondents $(41.5 \%$ vs. $26.5 \%, \mathrm{p}=0.03)$.

Conclusion Despite the advent of mobile connectivity, self-notification remains the most popular form of $\mathrm{PN}$ in our clinic attendees similar to the previous studies conducted in the United Kingdom. Respondents were also receptive to SMS PN, 